Behavior and Social Issues, 13, 33-50 (2004). (C) Jeffrey N. Weatherly and Andrew E. Brandt. Readers of this article may copy it without the copyright owner's permission, if the author and publisher are acknowledged in the copy and the copy is used for educational, not-for-profit purposes.

\title{
Participants' Sensitivity to Percentage Payback and Credit Value When Playing a Slot-Machine Simulation ${ }^{1}$
}

\author{
Jeffrey N. Weatherly \\ University of North Dakota \\ Andrew E. Brandt \\ Western Michigan University
}

\begin{abstract}
Legalized gambling has become both a major industry and concern in the United States, but little research from the behavior-analytic perspective has been done on the topic. The present study consisted of two experiments that had participants play a computer-simulated slot machine. The variables manipulated were the percentage payback rate (i.e., overall rate of reinforcement) and the amount of money the credits being wagered were worth (i.e., reinforcer magnitude). Experiment 1 investigated these variables using a between-groups design. Experiment 2 investigated them using a within-subjects design. Results from both experiments demonstrated that participants' gambling behavior did not vary as a function of payback percentage. Their behavior was, however, sensitive to credit value; overall, participants bet less when the credits were worth more. These findings have potential implications for why some people display "problem gambling." They will also hopefully promote research on a topic that has been largely ignored by the field of behavior analysis.

Key words: gambling, rate of reinforcement, magnitude of reinforcement, slot machine simulation
\end{abstract}

Because of its prevalence, legalized gambling has become a major social issue in the United States. Organized forms of gambling have been around since colonial times (Barker \& Britz, 2000), but they have only recently become widespread. By 1999 , at least some form of legalized gambling could be found in 48 states (MacLin, Dixon, \& Hayes, 1999). This spread has come at a price. Relatively recent evidence indicates that just over $1.5 \%$ of the adult population in the United States and Canada qualify as pathological gamblers (Shaffer, Hall, \& Vander Bilt, 1999). Although this percentage is small, in absolute terms it equates to a large number of people who suffer from financial losses, strained relationships, legal difficulties, and suicide (Petry, 2002). The increase in gambling has not gone unnoticed at the societal level. In fact, twice in the past 30 years (1976 \& 1996), the United States government has organized national-level commissions to assess the impact of gambling on society.

\footnotetext{
${ }^{1}$ Portions of the present study were completed in partial fulfillment of Andrew Brandt's senior honor's thesis. The authors thank Kelly Bishop and Melanie Hulst for their help in data collection. They also thank Jeffrey E. Holm and Cheryl Terrance for their statistical expertise. Correspondence regarding this manuscript should be addressed to Jeffrey N. Weatherly, Department of Psychology, University of North Dakota, Grand Forks, ND 58202-8380 (jeffrey_weatherly@und.nodak.edu).
} 
Despite its prevalence and the growing national attention, very little research has been conducted on gambling behavior from a behavior-analytic perspective. Skinner commented on the processes that contribute to and maintain gambling behavior, but did not conduct research on the topic (see Knapp, 1997). Others have since collected and reported empirical data (e.g., Dixon, Hayes, Rehfeldt, \& Ebbs, 1998; Lyons \& Ghezzi, 1995) as well as forwarded theoretical accounts from the behavioral perspective (Petry \& Roll, 2001; Rachlin, 1990). However, such attempts have been relatively rare. In short, although the idea that environmental contingencies influence gambling behavior has not been altogether neglected by the field as a whole (e.g., Dickerson \& Baron, 2000), the impact of behavior analysis has been small on a body of research on gambling that has become immense.

One potential reason for the dearth of research on gambling from a behavioranalytic perspective is that gambling behavior does not lend itself well to study. Studies in the area of the experimental analysis of behavior are typically conducted in controlled situations where the researcher can manipulate both the antecedents and consequences experienced by the subject. However, with gambling, such control is difficult (if not illegal). For instance, gambling involves a response-cost contingency in that the gambler can lose and thus actually leave the situation with less money than he or she came with. For ethical reasons, this scenario is difficult to mimic in a laboratory setting. Some researchers have proposed animal models of gambling (Kendall, 1987) to overcome such problems, but to date such models have not been pursued. Another difficulty in conducting research on gambling behavior is the inability to obtain the necessary equipment. For example, some states have laws that prohibit ownership of gambling devices except under certain conditions (e.g., the device is an antique/over 25 years old). Ironically, ownership of state-of-the-art devices is restricted to those in the gambling industry. However, even if one were to obtain such a device, it is typically not possible to control the consequences delivered by it (i.e., change the rate at which the machine pays off), which sound behavior-analytic research would require.

Modern technology has fortunately started to address this latter difficulty. For instance, MacLin et al. (1999) created a computer software program which simulates many of the characteristics of an actual slot machine, but also provides the researcher with a great deal of experimental control. Specifically, the program allows the researcher to dictate the symbols that will appear, the probability that those symbols will appear on the "win line," the combination of symbols that will be considered a "win" and how much each particular winning combination will pay, the number of credits staked to the participant, the number of credits the participant is allowed to bet per play, and the maximum number of times the participant is allowed to play. Because modern slot machines are video based (versus having actual reels inside the machine), this software also captures some realistic qualities of an actual slot machine (see MacLin et al., 1999, for a complete description).

With the control allowed by this slot-machine simulation, it becomes possible to begin studying gambling behavior from a behavior-analytic viewpoint. The 


\section{SLOT MACHINE SIMULATION}

present study did so by manipulating two variables that have long been known to influence behavior, reinforcement rate and reinforcer magnitude. These variables were studied across two experiments. Experiment 1 studied them using a betweengroups experimental design. Experiment 2 studied them using a within-subjects design.

Although a between-groups design is not the standard one used in the field of behavior analysis, it was employed in Experiment 1 for three reasons. First, it is the standard design within the general field of psychology. By using it, the results have an increased chance of being accepted by the field as a whole. Second, data collected from the present study can potentially serve as the foundation for future studies that will necessarily require the use of between-groups designs. For instance, future research will undoubtedly focus on whether the behavior of people who have been diagnosed as "problem gamblers" are differentially sensitive to certain contingencies relative to "normal" controls. Results from experiments like the present one will help to establish the behavior of "normal" controls. Third, by studying gambling behavior using both a between-groups and a within-subjects design (Experiment 2), it becomes possible to start identifying the mechanisms underlying gambling behavior. Both types of design have drawbacks to their use, ${ }^{2}$ but the drawbacks differ between them. Thus, if one finds the same pattern of results in both types of design when manipulating the same variables, then one can be reasonably assured that behavior is being controlled by those variables.

Regardless of research design, both experiments were attempts to assess participants' sensitivity to how often the slot-machine simulation paid off (i.e., percentage payback) and to how much the credits being used when playing the simulation were worth. ${ }^{3}$ These two factors, percentage payback ${ }^{4}$ and credit value, can be equated to overall reinforcer rate and magnitude, respectively. The standard finding in research on these variables has been that rates of behavior tend to increase with increases in both variables (e.g., see Mazur, 1998). However, because of myriad differences between a slot machine and the standard operant chamber, which has been used in the vast majority of studies manipulating these variables, it was possible that neither of these outcomes might be observed. For instance, although responses on a slot machine are reinforced on a random-ratio (RR) schedule, it is not a simple schedule. Because there are a number of different winning combinations of symbols, it would be more accurate to describe responses

\footnotetext{
${ }^{2}$ Between-groups designs compare averages across different groups of subjects, which may not accurately represent the behavior of any of the individual participants. Within-subjects designs measure an individual's behavior over repeated trials. Doing so gives rise to possibility of experience and/or order effects confounding the results.

${ }^{3}$ There are several ways to alter reinforcer magnitude in a gambling situation. The present study did so (only) by manipulating the monetary value of the credits. Other manipulations of reinforcer magnitude (e.g., altering the size of the payoffs for certain winning combinations) were not pursued by the present study, largely because such manipulations would have interacted with the other independent variable, percentage payback.

${ }^{4}$ Percentage payback refers to the average rate of return. So, for instance, if a player was playing a slot machine with a percentage payback of $95 \%$, the player would have, on average over an indefinite number of trials, 95 credits left for every 100 credits bet.
} 
being reinforced on conjoint RR schedules. The complexity of such schedules may influence participants' sensitivity to the overall payoff rate.

Furthermore, modern slot machines (and the simulation used here) pay wins by adding credits to the total number of credits accumulated by the participant rather than by delivering money immediately. Research has shown that, in such instances, participants' sensitivity to reinforcement may not be as great as it would be if reinforcement was tangible and collected immediately (e.g., Forzano \& Logue, 1994). Thus, because differences in magnitude may only truly occur at one point in the session (i.e., at the end, when the participant "cashes out"), it was possible that participants' behavior during the session would be little affected by differences in credit value.

Although the outcome of the present manipulations was uncertain, we made the following predictions for both experiments. First, slot-machine play (e.g., number of plays, total amount bet) would increase with increases in percentage payback. Second, slot-machine play would also increase with increases in credit value. Pursuing these predictions was deemed important for several reasons. For one, doing so allowed for an assessment of the software as a research tool. As noted above, the software itself is relatively new. Determining whether control over behavior can be established using it will help determine its usefulness in future research on gambling behavior. Second, the results could potentially take a large step in aiding our understanding of the factors that contribute to gambling behavior. By starting to establish how factors such as payback percentage and credit value influence gambling behavior, one can potentially start to formulate an overarching behavioral theory of (problem) gambling.

\section{EXPERIMENT 1}

\section{Method}

Participants. The participants were 63 college students (29 male, 34 female) who were recruited from the psychology department subject pool at the University of North Dakota. In order to participate, participants had to meet three criteria. First, they had to be 21 years of age or older. Second, they had to be capable of manipulating a computer mouse. Third, they could not display symptoms of problem gambling, assessed by the South Oaks Gambling Screen (SOGS; Lesieur \& Blume, 1987). Participants were compensated with course extra credit. Depending on which condition they experienced and their behavior when playing the slot-machine simulation, they also received monetary compensation.

Apparatus. The slot-machine simulation was loaded on to an IBM-compatible personal computer located in a room that measured approximately $3.7 \mathrm{~m}$ by $6.1 \mathrm{~m}$. The computer was located on a desk at which participants sat. The room also contained three other desks (with chairs) and computers which were not used in the experiment. Participants faced a wall which had a two-way mirror. The mirror was covered with paper and was not used in the experiment. Two exterior windows were located behind the participants (when they were facing the computer screen). 
The software was a custom version of that reported by MacLin et al. (1999). It varied in from the original in two major ways. First, the outcome for each individual trial (i.e., "play") was determined in advance by the researchers. Second, the payoff table was not shown on the computer screen. Rather, a sheet of paper that visually illustrated each winning combination of symbols and how much each combination paid was posted directly above the computer monitor. Otherwise, the features of the simulation were similar to those described by MacLin et al. (1999).

Procedure. Participants were run individually. Prior to the participant arriving, the researcher pseudo-randomly assigned the participant to a particular condition and programmed the software accordingly. When the participant entered the room, the person was directed to sit at the desk and was asked to read and sign an informed-consent form. Once informed consent was obtained, the researcher had the participant complete the SOGS. The SOGS consists of 16 questions designed to assess the participant's previous gambling activity. A score of five or greater on the SOGS has been shown to be a reliable indicator of pathological gambling behavior (Lesieur \& Blume, 1987). Upon completion, the researcher scored the SOGS. If the participant scored five or above, the researcher awarded the participant the course extra credit for participation and dismissed the person from the experiment. If the participant scored four or lower on the SOGS, the researcher read the person the following instructions:

For the next 15 minutes, you will be given the opportunity to play a computersimulated slot machine. This slot machine is programmed identically to those found in actual casinos. That is, each possible winning outcome is scheduled at a constant probability and each individual play is independent of the previous play. Two symbols will appear on the slot machine as you are playing: bells and cherries. The winning combinations of these symbols, as well as the payoffs for those combinations, appear directly above the computer. To win, the winning combination must appear on the middle row. You will be staked with 100 credits. The goal of each session is to end with as many credits as possible. You may bet one, two, or three credits per play by clicking on the appropriate button. You may quit at any time by clicking the "exit" button at the bottom of the screen. The session will end when a) you click "exit," b) you reach 0 credits, or c) 15 minutes have gone by. Do you have any questions?

If the participant had questions, they were answered by repeating the above instructions. For participants responding in sessions in which the credits were worth money, the sentences "Each credit is worth $\$ 0.01(\$ 0.10)$. Thus, you are being staked with $\$ 1.00$ ( $\$ 10.00$ ). You will be paid real U.S. cash money for the remaining credits at the end of the session." were inserted into the above instructions immediately subsequent to informing the participant that he or she had been staked with 100 credits. Regardless of condition, three bells in the middle row paid 16 credits. Three cherries paid 8 credits. Cherries in the first two spots and a blank in the third spot paid 4 credits. A cherry in the first spot and blanks in the second and third spots paid 2 credits. Blanks in all three positions paid 1 credit. 


\section{WEATHERLY \& BRANDT}

The above credit values were based on a one-credit bet. Those values were doubled and tripled when the participant bet two and three credits, respectively.

Participants then played the simulation until one of the above criteria was met. Upon completion, the participant was debriefed, given course extra credit and, depending on condition, paid for the total credits remaining at the end of the session, and dismissed from the experiment. Session length never exceeded 30 $\min$.

The experiment employed a 3 (percentage payback) by 3 (credit value) factorial design. The three percentage payback values were $75 \%, 83 \%$, and $95 \%$. These percentages were chosen because they ranged from a relatively poor to a relatively good, respectively, rate of return. Furthermore, it was possible to program these percentages by manipulating the probabilities of the three symbols appearing on the "win line," thus allowing the payoff for each winning combination to remain constant across conditions. For each payback percentage, a sequence of 150 outcomes was created by randomly sampling from a finite pool of symbols (a bell, cherry, or blank) to determine which would appear in each of the three positions on the middle row. Different payback percentages were created by altering the number of each type of symbol in the pool of symbols. A total of 150 outcomes was chosen because pilot work demonstrated that this amount exceeded the number of plays that could possibly occur in a 15 -min period. Each participant in a particular percentage-payback condition thus experienced the identical outcomes in the identical order (i.e., the same series of 150 outcomes). However, the actual percentage payback experienced by any one participant was determined not only by the condition to which the person had been assigned, but also by the number of trials the participant played and the amount of credits bet per trial across all of those trials. The second independent variable was credit value. The three levels of value were $\$ 0.00, \$ 0.01$, and $\$ 0.10$.

There were thus nine groups of participants. Each group had seven participants. Seven of the nine groups were made up of three male and four female participants. The remaining two groups had four male and three female participants.

Those participants playing for credits worth $\$ 0.01$ or $\$ 0.10$ were paid at the completion of the session for the credits they had remaining. Although it was not possible for participants to leave with less money than they had arrived with, it was possible for them to lose all the money they had been staked. It was also possible for them to finish the session with more money than they had been staked (i.e., to "win"). Lastly, participants were under no overt obligation to play the simulation. That is, they could immediately press the exit button without playing the simulation and, if they had been staked credits with a monetary value, simply receive the money they had been staked.

Data analysis. Although the software collected numerous measures during the session, the present study focused on the following dependent measures: the total number of trials the participant played during the session, the total number of credits bet across the session, and the total number of credits remaining at the end of the session. Because these measure were interrelated, they were initially 


\section{SLOT MACHINE SIMULATION}

analyzed using a multivariate analysis of variance (MANOVA), with payback percentage and credit value used as the grouping factors. If a significant effect was found in the multivariate analysis, then univariate analyses were conducted separately on each of the dependent measures to investigate the multivariate effect. When post hoc tests were required, Tukey tests were employed. Results were considered significant at $\mathrm{p}<.05$.

\section{Results}

Figure 1 presents the results from Experiment 1. Presented are the total number of trials per session (top graph), credits bet per session (middle graph), and number of credits left at the end of the session (bottom graph) as a function of credit value. The columns represent the mean for all participants responding at the $75 \%$ (solid bars), $83 \%$ (striped bars), and 95\% (dashed bars) percentage-payback rates. The error bars represent the standard error of the mean for each measure. Overall, the results in Figure 1 suggest that payback percentage had little influence on participants' behavior, although it did influence the number of credits they had remaining at the end of the session. The results also suggest that participants' behavior was sensitive to manipulations of credit value.

Results from the statistical analyses generally confirmed these impressions. Results from the MANOVA indicated a significant effect of percentage payback (Pillai's $=.636, \mathrm{~F}(6,106)=8.24, \mathrm{p}<.001)$ and of credit value (Pillai's $=.399, \mathrm{~F}(6$, $106)=4.41, \mathrm{p}<.001)$. The interaction between percentage payback and credit value, at the multivariate level, was not significant.

To investigate the influence of percentage payback, univariate tests were conducted for each dependent measure. The only significant effect of percentage payback was found for the number of credits left at the end of the session $(F(2,54)$ $=27.86, \mathrm{p}<.001)$. Post hoc tests revealed that participants had significantly more credits left when the percentage payback was $95 \%$ than when it was either $75 \%$ or $83 \%$. There was no difference in the number of credits left between participants in the $75 \%$ and $83 \%$ conditions.

Results of univariate tests also indicated a significant effect of credit value on the total number of trials per session $(\mathrm{F}(2,54)=4.04, \mathrm{p}<.023)$, total number of credits bet per session $(\mathrm{F}(2,54)=7.81, \mathrm{p}<.001)$, and total number of credits left at the end of the session $(\mathrm{F}(2,54)=7.05, \mathrm{p}<.002)$. Post hoc tests showed that, when credits were worthless (i.e., \$0.00), participants played significantly more trials than when each credit was worth $\$ 0.10$. Participants bet significantly more credits when the credits were worthless than when they were worth $\$ 0.01$ or $\$ 0.10$ each. Participants bet fewer credits when credits were worth $\$ 0.10$ than when they were worth $\$ 0.01$, but this difference fell short of statistical significance. Finally, participants had significantly more credits left at the end of the session when the credits were worth $\$ 0.01$ each than when they were worthless. They also had more credits left when the credits were worth $\$ 0.10$ than when they were worthless, but this difference fell short of significance. There was no significant difference in the number of credits left between participants in the $\$ 0.01$ and $\$ 0.10$ conditions. 
TRIALS PER SESSION
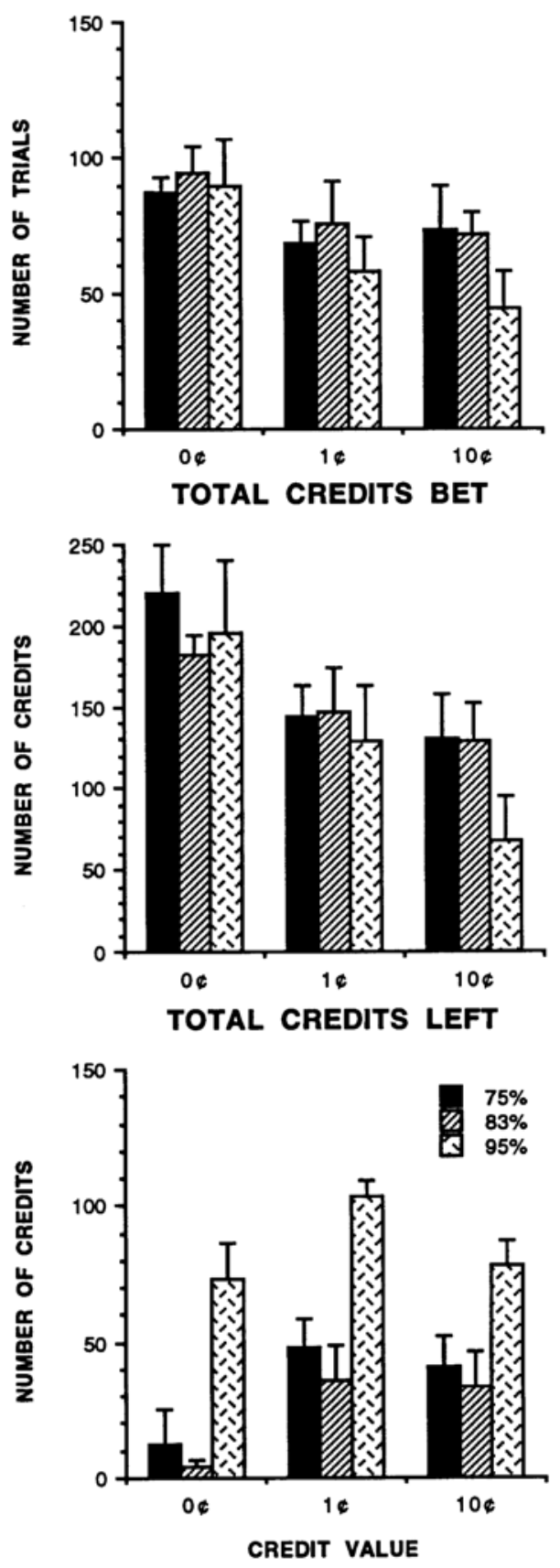

Figure 1. Presented are the mean number of trials per session, total credits bet per session, and total credits left at the end of the session for participants in each condition of Experiment 1. 


\section{Discussion}

The results of Experiment 1 paint an interesting, and counter-intuitive, picture. First, although years of operant research has indicated that rate of reinforcement is one of the primary determinants of the frequency of behavior, the present results indicate that participants' behavior was not sensitive to differences in payback percentage (the equivalent of overall rate of reinforcement). The only influence of percentage payback that was observed was on the number of credits participants had remaining at the end of the session. This particular outcome can be taken as evidence that percentage payback was actually manipulated. However, that manipulation had little effect on either the number of times participants played the simulation or the overall amount that they bet while playing.

Participants' behavior was sensitive to the different credit values, but this sensitivity was in the opposite direction than predicted. We originally predicted that rates of behavior would increase with increases in credit value (i.e., increases in reinforcer magnitude). But the opposite was observed. As credit value increased, the number of trials participants played before ending the session, and the number of credits they bet during the session, generally decreased. In short, as the credits increased in value, participants "gambling" decreased.

Although the present results suggest that, when participants played the slotmachine simulation, their behavior was governed more by credit value than by payback rate, the results should be interpreted cautiously due to the experimental design that was used. Because Experiment 1 employed a between-groups design, each participant only played the simulation one time (for a maximum of $15 \mathrm{~min}$ ). Under these conditions, it may not be surprising that credit value, and not payback percentage, had a greater influence over behavior. Participants were explicitly informed of the credit value and thus their behavior may have been rule governed (e.g., Hayes, Brownstein, Haas, \& Greenway, 1986; Lowe, Beasty, \& Bentall, 1983). Payback percentage, on the other hand, had to be ascertained through experience. It is possible that the present procedure simply failed to provide enough experience for participants to discriminate the overall payback percentage.

\section{EXPERIMENT 2}

Experiment 2 replicated the procedure of Experiment 1 with the one major exception that it employed a within-subjects design. Specifically, participants played the simulation a total of nine times, one time at each combination of the independent variables. As noted above, if similar results are obtained with a within-subjects design as with a between-groups design, then one gains confidence that the independent variables under study actually control behavior. Therefore, in Experiment 2 we were especially interested in pursuing the two major findings from Experiment 1. The first was the finding that participants were not sensitive to changes in overall rate of reinforcement. If this outcome was produced by the lack of experience given to the participants in Experiment 1, then Experiment 2 should produce such sensitivity because subjects were given extended experience. The 


\section{WEATHERLY \& BRANDT}

second was the finding that "gambling" (i.e., the number of trials per session $\&$ the total number of credits bet per session) decreased with increases in credit value. If credit value truly influences behavior, then similar findings should be observed in Experiment 2.

\section{Method}

Participants and Apparatus. The participants were eight university students (four male; four female) who were recruited and compensated as were those in Experiment 1. They had to meet the same standards for participation as in Experiment 1. The same equipment, software, and room were also used.

Procedure. The procedure used in Experiment 2 was identical to that used in Experiment 1 with the following exceptions. First, each of the eight participants responded in a total of nine sessions $(75 \%, 83 \%, \& 95 \%$ payback rates with credits worth $\$ 0.00, \$ 0.01, \& \$ 0.10$ each). Second, the SOGS was administered only prior to the initial session. Course extra credit was awarded only after the final session. Third, because each participant responded at each payback percentage on three different occasions, three separate 150-outcome sequences were created for each percentage-payback rate. This precaution was taken to ensure participants did not "memorize" the exact series of outcomes at each payback percentage. As in Experiment 1, the exact overall payback percentage experienced by the participant was determined not only by the outcomes programmed by the series, but also by how many trials the participant played and how much was bet on each trial.

As in Experiment 1, participants were read the (same) instructions prior to each session that included the appropriate statements regarding credit value for that particular condition. When participants played for credits that had monetary value, they were paid at the termination of each session for any credits they had remaining.

The order of conditions experienced by each participant was randomly determined. Which series of outcomes for each payback percentage were matched with which credit value was also randomly determined. Participants responded in a maximum of one session per day. For all participants, the time period necessary to complete all nine conditions never exceeded three weeks.

Data analysis. As in Experiment 1, the measures of interest were the number of trials played per session, the total number of credits bet per session, and the total number of credits remaining at the end of the session. Data were again analyzed in a multivariate fashion. However, because Experiment 2 employed a withinsubjects design, a doubly multivariate repeated-measures analysis was employed. Furthermore, because we were specifically interested in whether the significant effects observed in Experiment 1 were replicated in Experiment 2 (i.e., a priori predictions), certain univariate tests (e.g., on credit value) were pursued regardless of the outcome of the multivariate test. When post hoc tests were required, pairwise comparisons for repeated measures (i.e., correlated-sample t tests) were employed. Again, results were considered significant at $\mathrm{p}<.05$. 


\section{SLOT MACHINE SIMULATION}

\section{Results and Discussion}

Figures 2, 3, and 4 present the results from Experiment 2. Figure 2 presents the number of trials per session as a function of credit value. Each graph presents the results for an individual participant. The columns represents the $75 \%$ (solid bars), $83 \%$ (striped bars), and 95\% (checked bars) payback conditions. Figures 3 and 4 were identically constructed with the exception that Figure 3 presents the total number of credits bet per session and Figure 4 presents the total number of credits left per session.

A visual inspection of Figure 2 suggests that, although systematic changes in the number of trials played per session were evident across conditions for certain participants (e.g., participant 7), there was much variability across participants. Data on the number of credits bet per session were less variable. Although there

were some exceptions, participants generally displayed a decrease in the number of credits bet as a function of credit value. Systematic changes were also apparent as a function of percentage payback, but the direction of the change varied across participants. Finally, Figure 4 suggests that the number of credits remaining per session varied directly with payback percentage. An influence of credit value was apparent for some participants (e.g., participant 7), but less so for others.

The results from the statistical analyses tended to support these visual impressions. Results from the multivariate analysis indicated that the effect of percentage payback was significant (Pillai's $=1.024, \mathrm{~F}(6,26)=4.55, \mathrm{p}<.003$ ). Results from the univariate analyses indicated that only the number of credits left at the end of the session varied as a function of percentage payback $(\mathrm{F}(2,14)=$ $28.50, \mathrm{p}<.001)$. Participants ended the session with more credits in the $95 \%$ payback rate condition than either the $75 \%$ or $83 \%$ conditions. The number of credits left did not differ between the $75 \%$ and $83 \%$ conditions. Thus, as in Experiment 1, the only systematic influence of percentage payback was on the number of credits participants had remaining at the end of the session.

Unlike in Experiment 1, the multivariate effect of credit value was not significant. However, because of our a priori interest in determining whether the significant effects of credit value observed in Experiment 1 were present in Experiment 2, univariate analyses were pursued for the total number of trials per session and the total number of credits bet per session. A significant effect of credit value was observed for the total number of credits bet per session $(F(2,14)=4.45$, $\mathrm{p}<.032$ ). Comparisons of the different conditions showed that participants bet significantly more credits when the credits were worth $\$ 0.01$ than when they were worth $\$ 0.10$. Participants bet more credits when the credits were worthless than when they were worth $\$ 0.10$, but this difference did not reach statistical significance $(p<.053)$. The total number of trials per session did not vary as a function of credit value. Thus, the results of Experiment 2 did replicate those of Experiment 1 in that the total number of credits participants bet per session was influenced by credit value. Again, this influence tended toward an inverse relationship between credit value and amount bet. 

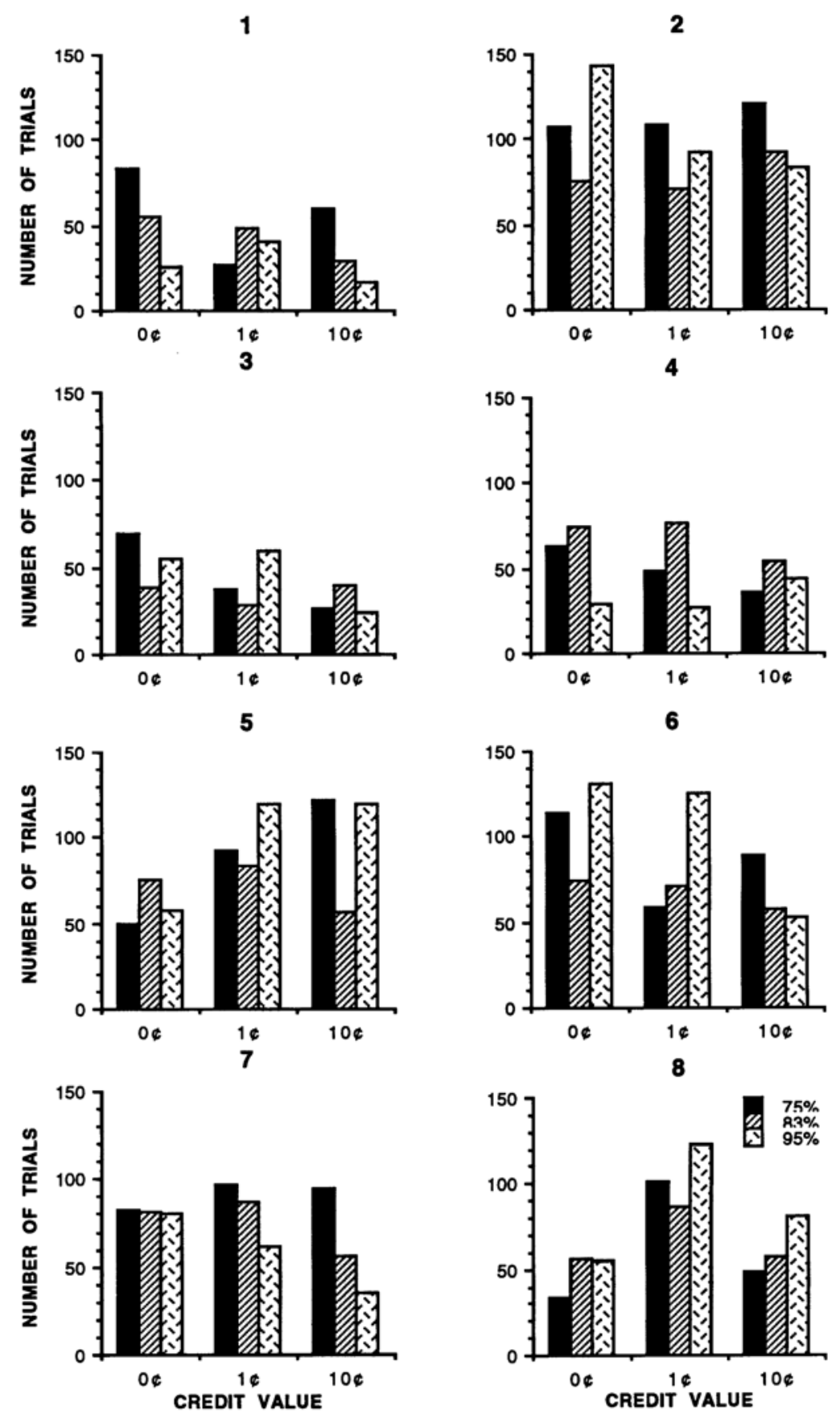

Figure 2. Presented are the total number of trials per session for each participant in each condition of Experiment 2. 

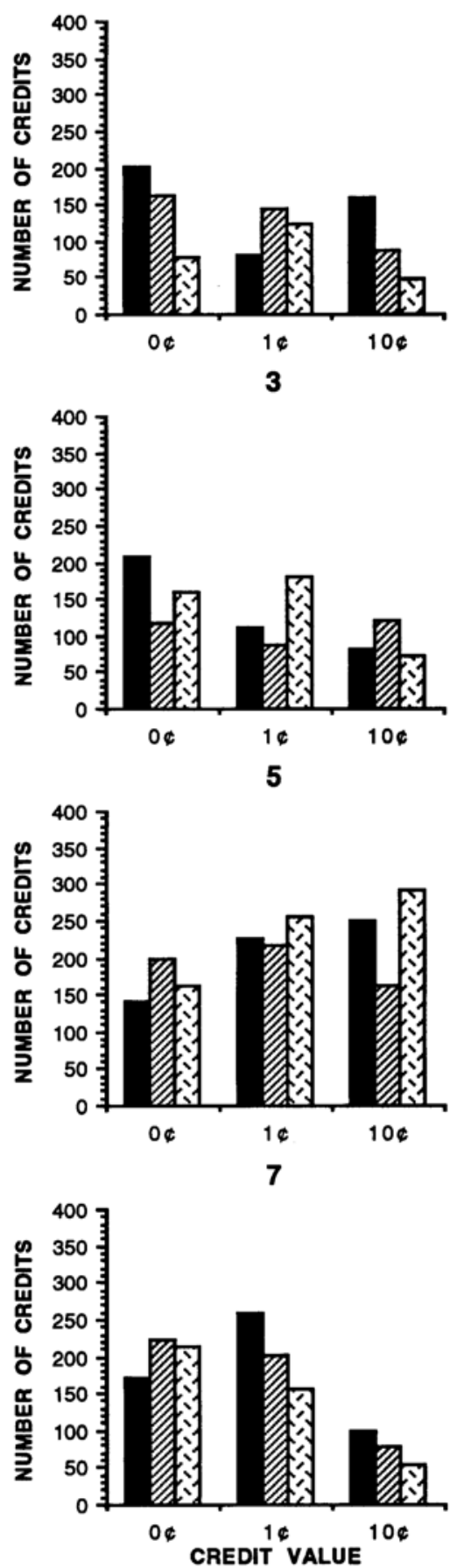

2
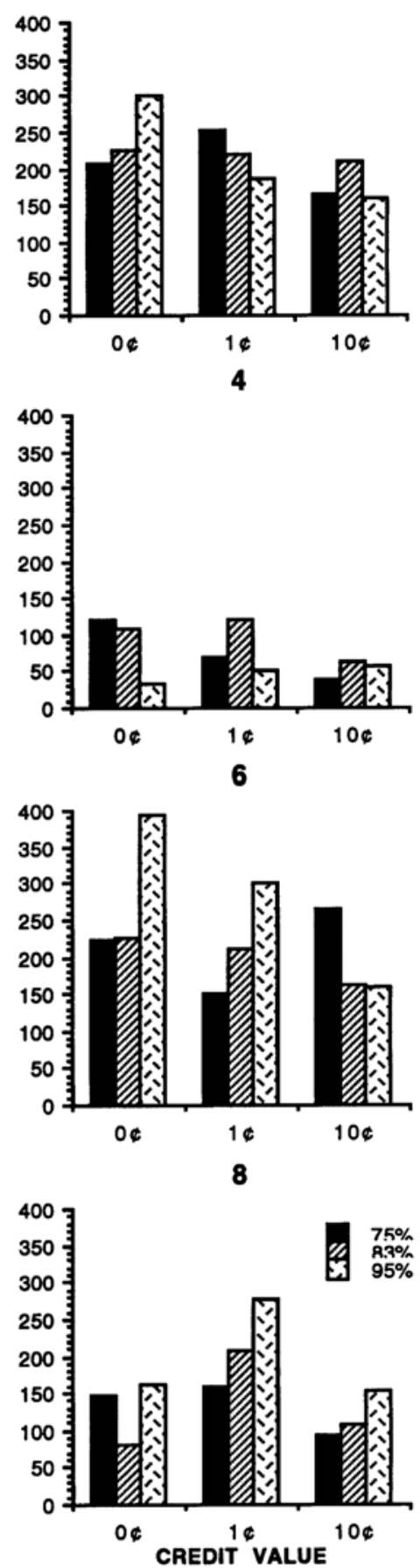

Figure 3. Presented are the total number of credits bet per session for each participant in each condition of Experiment 2. 

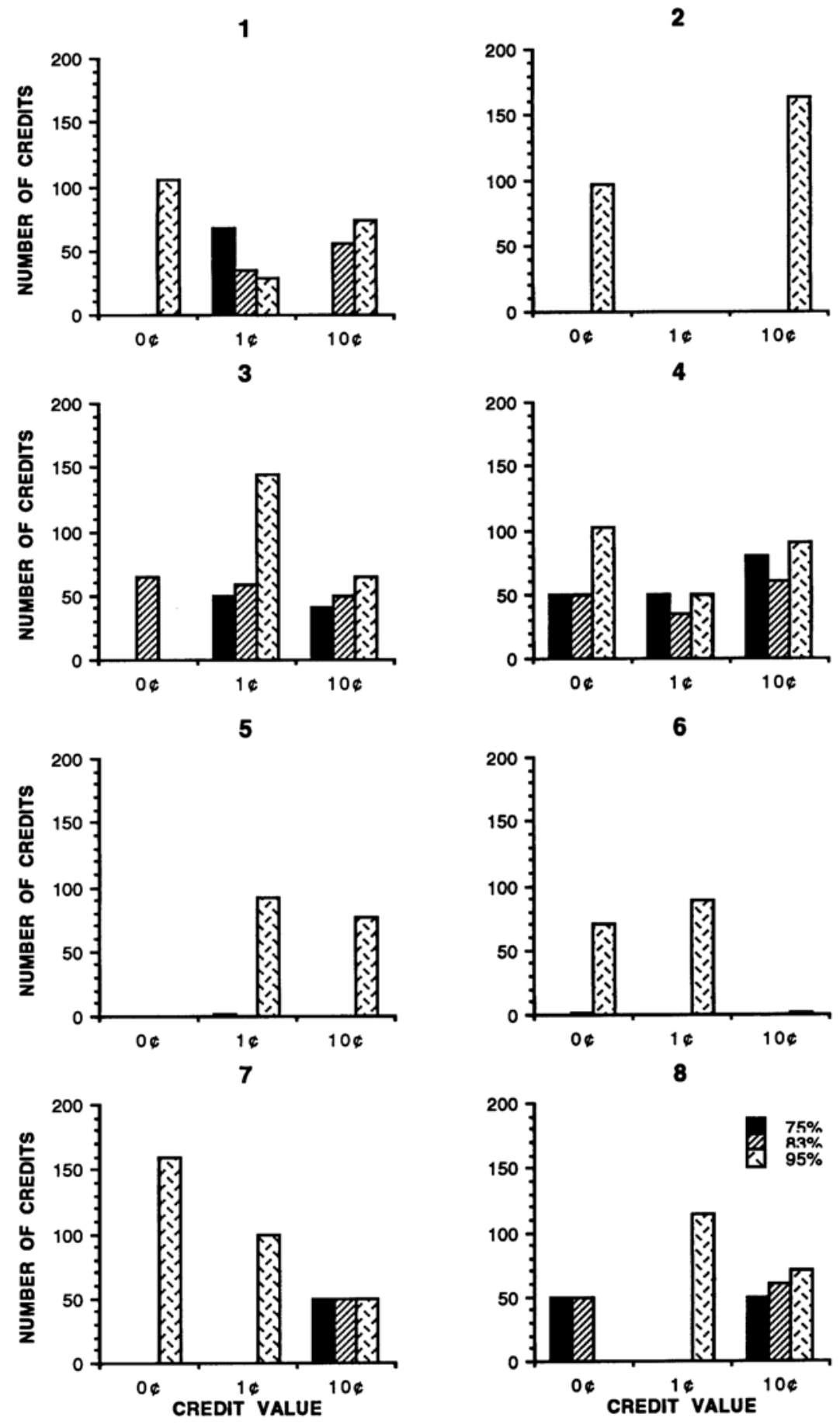

Figure 4. Presented are the total number of credits left per session for each participant in each condition of Experiment 2. 


\section{SLOT MACHINE SiMULATION}

\section{GENERAL DISCUSSION}

The present study was undertaken to determine whether a computer simulation of a slot machine could be used to study participants' sensitivity to the percentage payback scheduled by the simulation and/or by the amount of money each credit was worth. Experiment 1 employed a between-groups design to study these variables while Experiment 2 employed a within-subjects design. Both investigated payback percentages of $75 \%, 83 \%$, and $95 \%$ and credit values of $\$ 0.00, \$ 0.01$, and $\$ 0.10$. Although there were minor differences in results, the overall pattern of results was similar between experiments. Participants' play on the slot-machine simulation was not significantly altered by changes in payback percentage. Their behavior did vary, however, as a function of how much the credits were worth.

Percentage payback (i.e., the overall rate at which the simulation "paid off") can be equated to the overall rate of reinforcement, a major determinant of operant behavior. Behavior of participants in Experiment 1, however, did not change with changes in this variable. Because participants in Experiment 1 only played the simulation one time, and thus were given limited opportunity to determine the payback percentage, it was possible that the lack of effect of this variable was due to lack of experience. But the results of Experiment 2 question this idea. Experiment 2 employed a within-subjects design, exposing each participant to each of the three payback percentages on three different occasions. Despite the increased experience, percentage payback still did not significantly alter participants' play. It is also important to note that in neither experiment was the failure of different payback percentages to produce different gambling behavior due to a failure of the procedure to actually manipulate the payback rates. In both experiments, participants ended the session with significantly more credits at the highest percentage payback than at the lower percentages.

Given that overall rate of reinforcement is a major determinant of operant behavior, the question that arises is why the behavior of participants in the present study did not vary as a function of this variable? At least three, potentially related, explanations may account for this finding. First, it is possible that such sensitivity would have emerged if participants had been given extended experience with the payback percentages beyond that allowed in Experiment 2 (nine 15-min-maximum sessions). Second, it is possible that, because there were five different winning combinations of symbols, participants' behavior was sensitive to molecular differences in reinforcement rate rather than molar differences. In other words, because of the complexity of contingencies contributing to overall payback percentage, participants' behavior may have been more influenced by one contingency (e.g., winning the "jackpot") than by their overall rate of return. Third, it is possible that, again perhaps because of the complexity of the schedules of reinforcement involved, behavior when playing a slot machine is simply not sensitive to the differences in percentage payback.

The present data cannot delineate between these, or other, possibilities. Thus, future research will need to investigate why such insensitivity was found in the 
present study. It can be said, however, that regardless of which of the above explanations best explains the apparent insensitivity to overall percentage payback, the idea that there is such an insensitivity does not bode well for the gambling populace (and/or does bode well for the gaming industry). Obviously, if people gamble at a similar rate despite large differences in their overall payback percentage, then there is potential for huge losses when payback percentages are low. Even if sensitivity does appear with extensive experience, the loss of money that may occur while gaining that experience could be immense.

Although not the topic of the present study, the failure to find participants sensitive to changes in the overall payback rate has potential implications for the study of problem gambling. Intuitively, one might predict that problem or compulsive gamblers display such behavior because their sensitivity to the overall rate of reinforcement is lower than for non-problem gamblers. However, the present study found that the gambling of non-problem gamblers (according to the SOGS) did not differ with changes in payback percentage. The present results may thus suggest that, of the factors contributing to problem gambling, lack of sensitivity to percentage payback is not one of them. In fact, it would not be out of the realm of reason to expect that experienced gamblers, because of their experience, might actually be more sensitive to payback rate than non-experienced gamblers.

The implications of the present results related to credit value would seem to be more encouraging than those related to payback percentage. Experiment 1 found that, when credit value was increased, both the number of trials per session and the total credits bet across the session decreased. Experiment 2 replicated the finding that increases in credit value also led to a decrease in the total number of credits bet per session. In short, the more the credits were worth, the less participants gambled.

Although the present results cannot document exactly why participants' behavior was significantly altered by credit value but not payback percentage, it seems reasonable that this outcome occurred because credit value was simply a more salient variable than percentage payback. As noted above, behavior in regards to credit value may have been rule governed. Participants were overtly informed prior to each session what the credits were worth. Also, as they played the simulation, the credit counter continually informed them of the number of credits they possessed. No overt instructions were ever provided about payback percentage. Moreover, the information that was provided (i.e., the accumulation or loss of credits) was a less than perfect indicator (e.g., changes in total credits also occurred with changes in bet size).

Despite the decrease in amount bet with increases in credit value, it is intriguing to note that not a single participant "cashed out" before playing the simulation. That is, participants were informed prior to each session that they could end the session at any time. Thus, for participants in the $\$ 0.10$ conditions, ending the session before ever playing would have resulted in receiving $\$ 10.00$. But none did. Therefore, although it is accurate to say that participants gambled less as credit 
value increased, it is also accurate to say that the decrease in gambling was not complete (i.e., they all gambled).

Again, the present study was not designed to provide information on the behavior of problem gamblers, but the results pertaining to credit value may indirectly do so. The present study employed non-problem gamblers (according to the SOGS) and found that their gambling decreased as credit value increased. It seems reasonable to expect that a similar finding would not be obtained if studying the behavior of problem gamblers, thus potentially accounting for their "problem" behavior.

Ironically, the highest amount of "gambling" in the present study was observed when participants were playing with credits that were worthless. In other words, participants bet the most when what they were betting had no value. Such a finding would seem to make intuitive sense; participants in these conditions were not risking anything. However, this finding also highlights the reinforcing value of playing a slot machine (simulation). Participants playing for worthless credits also gained nothing by playing the simulation. Yet none of these participants terminated the session without playing. One implication of this finding is that games of chance, such as the one used here, have reinforcing consequences independent of the potential to win money.

As noted above, there have been few studies of gambling behavior from a behavior-analytic perspective, perhaps largely due to the inability to study such behavior using standard behavior-analytic designs. To this end, the software used in the present experiments (MacLin et al., 1999) may be a large step toward promoting such research. Its use produced interpretable and reliable results. Further research using this technology will ultimately determine its usefulness. Thus far, however, it has performed admirably.

Even with such promise, however, there will necessarily be a limit to the study of gambling in the laboratory. For ethical reasons, it will be difficult if not impossible to create the true response-cost contingency presented to the gambler. That is, although it may be ethical to allow participants to "lose" all they money that they have been staked, allowing them to actually lose money will most likely remain prohibited. Thus, there are procedural limitations. There may also be theoretical limitations. For instance, recent research has suggested that different people gamble for different reasons, with some gambling to gain something (i.e., money, excitement) while others gamble as an escape mechanisms (e.g., escape from boredom; Neighbors, Lostutter, Cronce, \& Larimer, 2002). Laboratory situations can be created to provide the former, but providing the latter may prove to be difficult.

In closing, it is our hope that the dissemination of useful software and research reports such as the present study are the initial commitments by behavior analysts to study gambling behavior. Gambling has become a major industry, and a major concern, in the United States and it is time that behavior analysis properly addressed it. The recent formation of a special interest group on gambling behavior within the Association for Behavior Analysis hopefully signifies that behavior analysts are about to weigh in. 


\section{REFERENCES}

Barker, T., \& Britz, M. (2000). Jokers wild: Legalized gambling in the twenty-first century. Westport, CT: Prager.

Dickerson, M., \& Baron, E. (2000). Contemporary issues and future directions for research into pathological gambling. Addiction, 95, 1145-1159. http://dx.doi.org/10.1080/09652140050111087

Dixon, M. R., Hayes, L. J., Rehfeldt, R. A., \& Ebbs, R. E. (1998). A possible adjusting procedure for studying outcomes of risk-taking. Psychological Reports, 82, 1047-1050. http://dx.doi.org/10.2466/pr0.1998.82.3.1047

Forzano, L. B., \& Logue, A. W. (1994). Self-control in adult humans: Comparison of qualitatively different reinforcers. Learning and Motivation, 25, 65-82. http://dx.doi.org/10.1006/lmot.1994.1004

Hayes, S. C., Brownstein, A. J., Haas, J. R., \& Greenway, D. E. (1986). Instructions, multiple schedules, and extinction: Distinguishing rule-governed behavior from schedule-controlled behavior. Journal of the Experimental Analysis of Behavior, 46, 137-147. http://dx.doi.org/10.1901/jeab.1986.46-137

Kendall, S. B. (1987). An animal analogue of gambling. The Psychological Record, 37, 247-256.

Knapp, T. J. (1997). Behaviorism and public policy: B.F. Skinner's views on gambling. Behavior and Social Issues, 7, 129-139. http://dx.doi.org/10.5210/bsi.v7i2.311

Lesieur, H. R., \& Blume, S. B. (1987). The south oaks gambling screen (SOGS): A new instrument for the identification of pathological gamblers. American Journal of Psychiatry, 144, 1184-1188.

Lowe, C. F., Beasty, A., \& Bentall, R. P. (1983). The role of verbal behavior in human learning: Infant performance on fixed-interval schedules. Journal of the Experimental Analysis of Behavior, 39, 157-164. http://dx.doi.org/10.1901/jeab.1983.39-157

Lyons, C. A., \& Ghezzi, P. M. (1995). Wagering on a large scale: Relationships between public gambling and game manipulation in two state lotteries. Journal of Applied Behavior Analysis, 28, 127-137. http://dx.doi.org/10.1901/jaba.1995.28-127

MacLin, O. H., Dixon, M. R., \& Hayes, L. J. (1999). A computerized slot machine simulation to investigate the variables involved in gambling behavior. Behavior Research Methods, Instruments, \& Computers, 31, 731-734. http://dx.doi.org/10.3758/BF03200755

Mazur, J. E. (1998). Learning and behavior (4th ed.). Upper Saddle River, NJ: Prentice Hall.

Neighbors, C., Lostutter, T. W., Cronce, J. M., \& Larimer, M. E. (2002). Exploring college student gambling motivation. Journal of Gambling Studies, 18, 361370. http://dx.doi.org/10.1023/A:1021065116500

Petry, N. M. (2002). A comparison of young, middle-aged, and older adult treatment seeking pathological gamblers. The Gerontologist, 42, 92-99. http://dx.doi.org/10.1093/geront/42.1.92

Petry, N. M., \& Roll, J. M. (2001). A behavioral approach to understanding and treating pathological gambling. Seminars in Clinical Neuropsychology, 6, 177-183. http://dx.doi.org/10.1053/scnp.2001.22920

Rachlin, H. (1990). Why do people gamble and keep gambling despite heavy losses? Psychological Science, 1, 294-297. http://dx.doi.org/10.1111/j.14679280.1990.tb00220.x

Shaffer, H. J., Hall, M. N., \& Vander Bilt, J. (1999). Estimating the prevalence of disordered gambling in the United States and Canada: A research synthesis. 
WEATHERLY \& BRANDT

American Journal of Public Health, 89, 1369-1376.

http://dx.doi.org/10.2105/AJPH.89.9.1369 